\title{
Ajustes de funções de distribuição de probabilidade à radiação solar global no Estado do Rio Grande do Sul
}

\author{
Alberto Cargnelutti Filho ${ }^{(1)}$, Ronaldo Matzenauer( ${ }^{(1)}$ e Júlio Kuhn da Trindade ${ }^{(2)}$
}

\begin{abstract}
(1)Fundação Estadual de Pesquisa Agropecuária, Rua Gonçalves Dias, 570, Bairro Menino Deus, CEP 90130-060 Porto Alegre, RS. E-mail: alberto-cargnelutti@fepagro.rs.gov.br, ronaldo-matzenauer@fepagro.rs.gov.br(2)Universidade Federal do Rio Grande do Sul, Av. Bento Gonçalves, 7712, Bairro Agronomia, CEP 91501-970 Porto Alegre, RS. E-mail: catrindade@ig.com.br
\end{abstract}

\begin{abstract}
Resumo - O objetivo deste trabalho foi verificar o ajuste das séries de dados de radiação solar global média decendial, de 22 municípios do Estado do Rio Grande do Sul, às funções de distribuições de probabilidade normal, log-normal, gama, gumbel e weibull. Aplicou-se o teste de aderência de Kolmogorov-Smirnov, nas 792 séries de dados (22 municípios x 36 decêndios) de radiação solar global média decendial, para verificar o ajuste dos dados às distribuições normal, log-normal, gama, gumbel e weibull, totalizando 3.960 testes. Os dados decendiais de radiação solar global média se ajustam às funções de distribuições de probabilidade normal, lognormal, gama, gumbel e weibull, e apresentam melhor ajuste à função de distribuição de probabilidade normal.
\end{abstract}

Termos para indexação: modelagem, redução de riscos.

\section{Adjustments of probability distribution functions to global solar radiation in Rio Grande do Sul State}

\begin{abstract}
The objective of this work was to verify the adjustment of data series for average global solar radiation to the normal, log-normal, gamma, gumbel and weibull probability distribution functions. Data were collected from 22 cities in Rio Grande do Sul State, Brazil. The Kolmogorov-Smirnov test was applied in the 792 series of data (22 localities x 36 periods of ten days) of average global solar radiation to verify the adjustment of the data to the normal, log-normal, gamma, gumbel and weibull probability distribution functions, totalizing 3,960 tests. The data of average global solar radiation adjust to the normal, log-normal, gamma, gumbel and weibull probability distribution functions, and present a better adjustment to the normal probability function.
\end{abstract}

Index terms: modelling, risk reduction.

\section{Introdução}

O conhecimento do comportamento da precipitação pluvial, temperatura e umidade relativa do ar, evaporação, direção e velocidade do vento, radiação solar global, ocorrência de orvalho, nevoeiro, granizo, geada e neve, entre outros, é um importante instrumento na tomada de decisões relacionadas às atividades agropecuárias. Entre essas variáveis climáticas, a radiação solar global, definida como o total de energia emitida pelo sol, que incide sobre a superfície terrestre, com comprimento de onda compreendido entre 150 e $4.000 \mathrm{~nm}$ (Rosenberg, 1974; Slater, 1980), é fundamental, principalmente em relação às atividades agropecuárias.

A simples visualização dos dados amostrais de uma variável em um histograma de freqüência é insuficiente para inferir, entre as diversas funções de distribuição de probabilidade conhecidas, a que melhor se ajusta aos dados em estudo. Portanto, faz-se necessário o uso de testes de aderência para verificar se a distribuição de probabilidade dos dados de uma variável em análise pode ser estudada por uma função de distribuição de probabilidade conhecida.

Existem diversas funções de distribuições de probabilidade para variáveis aleatórias discretas e contínuas. Entre as que se ajustam a dados discretos estão a bernoulli, binomial, binomial negativa, hipergeométrica, geométrica e poisson. Já as distribuições uniforme, normal, log-normal, gama, valores extremos ou gumbel, weibull, exponencial, beta, qui-quadrado, $t$ de Student, F de Snedecor, entre outras, podem ser ajustadas a série de dados amostrais de variáveis aleatórias contínuas. Estudos de ajustes de função de distribuição de probabilidade ou estimativas de probabilidade usando funções 
de distribuição de probabilidade teóricas em relação a variáveis climáticas, como precipitação pluvial (Berlato, 1987; Assad \& Castro, 1991; Assis, 1991, 1993; Castro, 1996; Ávila et al., 1996; Botelho \& Morais, 1999; Catalunha et al., 2002), temperatura do ar (Mota et al., 1999; Buriol et al., 2000b, 2000c) e radiação solar (Buriol et al., 2000a, 2001) têm sido desenvolvidos, enfatizando os benefícios no planejamento de atividades que minimizem riscos climáticos.

Testes de aderência, como o qui-quadrado, Kolmogorov-Smirnov, Lilliefors, Shapiro-Wilk, Cramervon Mises (Campos, 1983; Assis et al., 1996; Morettin \& Bussab, 2004), servem para comparar as probabilidades empíricas de uma variável com as probabilidades teóricas estimadas pela função de distribuição em teste, verificando se os valores da amostra podem razoavelmente ser considerados como provenientes de uma população com aquela distribuição teórica. Nos testes de aderência, a hipótese nula $\left(\mathrm{H}_{0}\right)$ admite que a distribuição seja a especificada (normal, log-normal, gama e outras), com os seus parâmetros estimados com base nos dados amostrais (Assis et al., 1996; Catalunha et al., 2002). Existem diferenças entre os testes de aderência, quanto à probabilidade de rejeitar $\mathrm{H}_{0}$, quando efetivamente $\mathrm{H}_{0}$ é falsa ( 1 - $\beta$ ). Os testes de quiquadrado e de Kolmogorov-Smirnov são amplamente utilizados (Assis et al, 1996), sendo o primeiro, normalmente, mais eficaz que o segundo (Catalunha et al., 2002). Já o teste de Lilliefors (Campos, 1983) é mais eficaz que o teste de Kolmogorov-Smirnov, porém é específico para verificar a aderência dos dados amostrais à distribuição normal. Os testes de Shapiro-Wilk e Cramer-von Mises também diferenciam-se quanto à sua eficácia.

O teste de aderência de qui-quadrado apresenta limitações. Por exemplo, a freqüência de uma classe não pode ser inferior a cinco e os dados são agrupados em classes perdendo informações, o que não ocorre no teste de Kolmogorov-Smirnov, que além de poder ser realizado com os dados agrupados, pode também ser realizado com os dados isoladamente, sendo normalmente mais eficiente que o qui-quadrado em pequenas amostras, ou seja, menos de 30 observações (Campos, 1983). O teste de Kolmogorov-Smirnov é baseado no módulo da maior diferença entre a probabilidade observada e a estimada, que é comparada com um valor tabelado de acordo com o número de observações da série sob teste (Catalunha et al., 2002).

Pesq. agropec. bras., Brasília, v.39, n.12, p.1157-1166, dez. 2004
O objetivo deste trabalho foi verificar o ajuste das séries de dados de radiação solar global média decendial de 22 municípios do Estado do Rio Grande do Sul às funções de distribuições de probabilidade normal, lognormal, gama, gumbel e weibull.

\section{Material e Métodos}

Os dados de radiação solar global das 22 estações meteorológicas, localizadas em 22 municípios do Estado do Rio Grande do Sul, foram obtidos no Banco de Dados do Laboratório de Agrometeorologia, da Fundação Estadual de Pesquisa Agropecuária Fepagro/SCT-RS (Tabela 1).

Os dados de radiação solar global foram coletados no período de 1956 a 2003, totalizando 47 anos de observações. Em cada município, com os dados diários de radiação solar global, estimou-se a média decendial dos 36 decêndios do ano (1ํ decêndio de

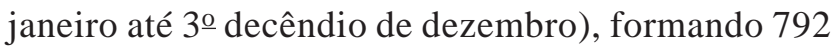
séries temporais (22 municípios x 36 decêndios), com número diferenciado de anos de observações em cada série em função da disponibilidade dos dados meteorológicos (Tabela 1).

Aplicou-se o teste de aderência de KolmogorovSmirnov (Campos, 1983; Assis et al., 1996) nas 792 séries de dados de radiação solar global média decendial, para verificar o ajuste dos dados às distribuições normal, log-normal, gama, gumbel e weibull (Assis et al., 1996; Morettin \& Bussab, 2004; Spiegel et al., 2004), totalizando 3.960 testes (792 séries de dados x 5 distribuições).

A partir das coordenadas geográficas das estações meteorológicas dos 22 municípios, realizou-se a interpolação de isolinhas da radiação solar global média decendial de cada mês e decêndio, com o auxílio do aplicativo Surfer 7.0.

\section{Resultados e Discussão}

O valor da estatística D máximo do teste de aderência de Kolmogorov-Smirnov (Campos, 1983; Assis et al., 1996; Morettin \& Bussab, 2004) informa a máxima distância entre as probabilidades empíricas e as teóricas obtidas sob a função de distribuição de probabilidade em teste. Assim, menores valores da estatística fornecem maiores valores de p-valor e, conseqüentemente, maior evidência de não-rejeição da hipótese nula $\left(\mathrm{H}_{0}\right)$, 
ou seja, maior aderência dos dados à distribuição em teste.

Independentemente da função de distribuição de probabilidade (normal, log-normal, gama, gumbel e weibull), 3.691 (93,21\%), dos 3.960 casos analisados (cinco distribuições x 22 municípios x 36 decêndios), se ajustaram às funções de distribuições de probabilidades testadas com p-valor $\geq 0,20$, ou seja, com boa aderência (Tabela 2). No nível de significância do teste de 0,05 , ou seja, com probabilidade de um erro tipo I de 0,05, o número de séries de dados que não rejeitaram $\mathrm{H}_{0}$, isto é, os dados que se ajustam à distribuição em teste, elevou-se para 3.914 (98,84\%). Portanto, apenas $1,16 \%$ dos casos não aderiram a nenhuma das distribuições neste nível de significância. Assim, pode-se inferir que entre as cinco distribuições testadas, os parâmetros de qualquer uma delas poderiam ser utilizados para representar o comportamento da radiação solar global média decendial. Porém, a obtenção da estimativa dos parâmetros dessas distribuições e a estimativa das probabilidades diferem quanto ao grau de dificuldade. Então, convém verificar qual das funções estudadas tem o melhor ajuste, podendo a mesma coincidir com uma distribuição que apresente menor dificuldade de obtenção dos parâmetros e ainda, facilidade nas estimativas de probabilidades.

Entre as cinco funções de distribuições de probabilidades testadas na classe de $p$-valor $\geq 0,20$, a distribuição normal apresentou o maior número de aderências (782 séries de dados), ou seja, apenas dez séries de dados não se ajustaram à distribuição normal, em nível de significância de 0,20. No entanto, os dez casos se ajustaram com $p$-valor $\geq 0,10$, eviden-

Tabela 1. Coordenadas geográficas das estações meteorológicas localizadas em 22 municípios do Estado do Rio Grande do Sul, período de coleta dos dados de radiação solar global média decendial e número de observações (mínimo, máximo e médio) entre as 36 séries (decêndios) de dados em cada município.

\begin{tabular}{|c|c|c|c|c|c|c|c|}
\hline \multirow[t]{2}{*}{ Município } & \multicolumn{3}{|c|}{ Coordenadas geográficas ${ }^{(1)}$} & \multirow[t]{2}{*}{ Período } & \multicolumn{3}{|c|}{ Número de observações } \\
\hline & Altitude (m) & Latitude (S) & Longitude (W) & & Mínimo & Máximo & Médio \\
\hline Alegrete & 96 & $29^{\circ} 46^{\prime} 59^{\prime \prime}$ & $55^{\circ} 46 ' 59^{\prime \prime}$ & $1968-2001$ & 15 & 20 & 17 \\
\hline Cachoeirinha & 4 & $29^{\circ} 57^{\prime} 36^{\prime \prime}$ & $51^{\circ} 04^{\prime} 22^{\prime \prime}$ & 1975-2002 & 26 & 27 & 27 \\
\hline Caxias do Sul & 787 & $29^{\circ} 10^{\prime} 25^{\prime \prime}$ & $51^{\circ} 12^{\prime} 21^{\prime \prime}$ & $1987-2003$ & 16 & 17 & 16 \\
\hline Cruz Alta & 473 & $28^{\circ} 38^{\prime} 21^{\prime \prime}$ & $53^{\circ} 364^{\prime \prime}$ & 1974-1998 & 21 & 24 & 23 \\
\hline Encruzilhada do Sul & 420 & $30^{\circ} 32^{\prime} 35^{\prime \prime}$ & $52^{\circ} 31^{\prime} 20^{\prime \prime}$ & 1958-1999 & 33 & 37 & 35 \\
\hline Erechim & 760 & $27^{\circ} 37^{\prime} 46^{\prime \prime}$ & $52^{\circ} 16^{\prime} 33^{\prime \prime}$ & 1966-1991 & 20 & 25 & 23 \\
\hline Farroupilha & 702 & $29^{\circ} 14^{\prime} 30^{\prime \prime}$ & $51^{\circ} 26^{\prime} 20^{\prime \prime}$ & 1963-1996 & 27 & 30 & 29 \\
\hline Ijuí & 448 & $28^{\circ} 23^{\prime} 17^{\prime \prime}$ & $53^{\circ} 54^{\prime} 50^{\prime \prime}$ & $1963-1990$ & 20 & 27 & 25 \\
\hline Júlio de Castilhos & 514 & $29^{\circ} 13^{\prime} 26^{\prime \prime}$ & $53^{\circ} 40^{\prime} 45^{\prime \prime}$ & 1956-1996 & 38 & 41 & 39 \\
\hline Santana do Livramento & 210 & $30^{\circ} 53^{\prime} 18^{\prime \prime}$ & $55^{\circ} 31^{\prime} 56^{\prime \prime}$ & $1965-1981$ & 13 & 16 & 15 \\
\hline Maquiné & 32 & $29^{\circ} 40^{\prime} 49^{\prime \prime}$ & $50^{\circ} 13^{\prime} 56^{\prime \prime}$ & 1957-1997 & 35 & 38 & 37 \\
\hline Passo Fundo & 709 & $28^{\circ} 15^{\prime} 41^{\prime \prime}$ & $52^{\circ} 24^{\prime} 45^{\prime \prime}$ & $1961-2003$ & 43 & 43 & 43 \\
\hline Quaraí & 100 & $30^{\circ} 23^{\prime} 17^{\prime \prime}$ & $56^{\circ} 26^{\prime} 53^{\prime \prime}$ & 1966-2000 & 29 & 33 & 31 \\
\hline Rio Grande & 15 & $32^{\circ} 01^{\prime} 44^{\prime \prime}$ & $52^{\circ} 15^{\prime} 37^{\prime \prime}$ & 1956-1997 & 30 & 35 & 33 \\
\hline Santa Maria & 95 & $29^{\circ} 41^{\prime} 25^{\prime \prime}$ & $53^{\circ} 48^{\prime} 42^{\prime \prime}$ & $1965-1998$ & 29 & 33 & 31 \\
\hline Santa Rosa & 273 & $27^{\circ} 51^{\prime} 50^{\prime \prime}$ & $54^{\circ} 29^{\prime} 03^{\prime \prime}$ & 1975-1999 & 21 & 24 & 22 \\
\hline São Borja & 99 & $28^{\circ} 39^{\prime} 44^{\prime \prime}$ & $56^{\circ} 00^{\prime} 15^{\prime \prime}$ & $1956-2000$ & 35 & 41 & 38 \\
\hline São Gabriel & 109 & $30^{\circ} 27^{\prime} 27^{\prime \prime}$ & $54^{\circ} 19^{\prime} 01^{\prime \prime}$ & $1963-2000$ & 31 & 37 & 35 \\
\hline Taquari & 76 & $29^{\circ} 48^{\prime} 15^{\prime \prime}$ & $51^{\circ} 49^{\prime} 30^{\prime \prime}$ & $1963-2000$ & 35 & 38 & 37 \\
\hline Uruguaiana & 74 & $29^{\circ} 45^{\prime} 23^{\prime \prime}$ & $57^{\circ} 05^{\prime} 12^{\prime \prime}$ & 1963-1991 & 20 & 24 & 22 \\
\hline Vacaria & 955 & $28^{\circ} 30^{\prime} 09^{\prime \prime}$ & $50^{\circ} 56^{\prime} 12^{\prime \prime}$ & 1966-1990 & 19 & 24 & 22 \\
\hline Veranópolis & 705 & $28^{\circ} 56^{\prime} 14^{\prime \prime}$ & $51^{\circ} 33^{\prime} 11^{\prime \prime}$ & 1956-1999 & 29 & 32 & 30 \\
\hline Todos os municípios & & & & & 13 & 43 & 29 \\
\hline
\end{tabular}

${ }^{(1)}$ Fonte: Instituto de Pesquisas Agronômicas (1989). 
ciando boa aderência dos dados a esta distribuição. Em outro extremo, a distribuição weibull apresentou os menores índices de aderência. Esta distribuição apontou 41 dos 46 casos com p-valor $<0,05$. As demais distribuições (log-normal, gama e gumbel) estão em situações intermediárias (Tabela 3).

O teste de aderência de Kolmogorov-Smirnov revelou a distribuição normal como a mais adequada ao estudo para representar radiação solar global média decendial, bastando estimar os parâmetros desta distribuição (média e desvio-padrão) para os cálculos de probabilidade dentro de limites de intervalos desejados.

As estimativas dos parâmetros média $(\hat{\mu})$ e desviopadrão $(\hat{\sigma})$ da radiação solar global média decendial em cada decêndio e município (Tabela 4) possibilitam estimar as probabilidades acima ou abaixo de qualquer valor de radiação solar global média decendial, visando minimização de riscos. Assim, a probabilidade da radia- ção solar global média decendial ser menor ou igual a $511 \mathrm{cal} \mathrm{cm}^{-2} \mathrm{dia}^{-1}$ no primeiro decêndio de janeiro no município de Alegrete é de 50\%.

De maneira geral, a radiação solar global média decendial apresentou maiores valores no primeiro e último mês do ano, ocorrendo diminuição nos meses de junho e julho. Resultado semelhante foi encontrado por Bergamaschi et al. (2003) em Eldorado do Sul, RS. Isto ocorreu por causa do solstício de verão que acontece na segunda quinzena de dezembro, com redução gradativa da insolação e aumento da declinação solar até o solstício de inverno, na segunda quinzena de junho.

De modo geral, na primavera e no verão, ocorreu um aumento da radiação solar global média decendial no sentido Nordeste-Sudoeste e nos meses de maio, junho, julho e agosto uma diminuição no sentido Norte-Sul. Já nos meses de março e abril, a maior radiação solar global média decendial ocorreu na Região Central do Rio Grande do Sul (Figuras 1, 2 e 3).

Tabela 2. Frequência absoluta $\left(f_{i}\right)$, frequência acumulada $\left(F_{i}\right)$, frequência relativa absoluta $\left(\mathrm{fr}_{\mathrm{i}}\right)$ e frequência relativa acumulada $\left(\mathrm{Fr}_{\mathrm{i}}\right)$ de ajustes das 792 séries de dados de radiação solar global média decendial às funções de distribuições de probabilidade normal, log-normal, gama, gumbel e weibull em 22 municípios do Estado do Rio Grande do Sul, em cada classe de p-valor, pelo teste de aderência de Kolmogorov-Smirnov.

\begin{tabular}{lrrrr}
\hline $\mathrm{p}$-valor & $\mathrm{f}_{\mathrm{i}}$ & $\mathrm{F}_{\mathrm{i}}$ & $\mathrm{fr}_{\mathrm{i}}(\%)$ & $\mathrm{Fr}_{\mathrm{i}}(\%)$ \\
\hline $\mathrm{p}<0,01$ & 18 & 18 & 0,45 & 0,45 \\
$\mathrm{p}<0,05$ & 28 & 46 & 0,71 & 1,16 \\
$\mathrm{p}<0,10$ & 61 & 107 & 1,54 & 2,70 \\
$\mathrm{p}<0,15$ & 76 & 183 & 1,92 & 4,62 \\
$\mathrm{p}<0,20$ & 86 & 269 & 2,17 & 6,79 \\
$\mathrm{p} \geq 0,20$ & 3.691 & 3.960 & 93,21 & 100,00 \\
\hline
\end{tabular}

Tabela 3. Frequência absoluta $\left(\mathrm{f}_{\mathrm{i}}\right)$ e frequência relativa absoluta $\left(\mathrm{fr}_{\mathrm{i}}\right)$ de casos em que os dados de 792 séries de radiação solar global média decendial se ajustaram às funções de distribuições de probabilidade normal, log-normal, gama, gumbel e weibull, em 22 municípios do Estado do Rio Grande do Sul, em cada classe de p-valor pelo teste de aderência de Kolmogorov-Smirnov.

\begin{tabular}{|c|c|c|c|c|c|c|c|c|c|c|}
\hline \multirow[t]{2}{*}{$\mathrm{p}$-valor } & \multicolumn{2}{|c|}{ Normal } & \multicolumn{2}{|c|}{ Log-normal } & \multicolumn{2}{|c|}{ Gama } & \multicolumn{2}{|c|}{ Gumbel } & \multicolumn{2}{|c|}{ Weibull } \\
\hline & $f_{i}$ & $\mathrm{fr}_{\mathrm{i}}$ & $f_{i}$ & $\mathrm{fr}_{\mathrm{i}}$ & $f_{i}$ & $\mathrm{fr}_{\mathrm{i}}$ & $f_{i}$ & $\mathrm{fr}_{\mathrm{i}}$ & $\mathrm{f}_{\mathrm{i}}$ & $\mathrm{fr}_{\mathrm{i}}$ \\
\hline $\mathrm{p}<0,01$ & 0 & 0,00 & 0 & 0,00 & 0 & 0,00 & 0 & 0,00 & 18 & 2,27 \\
\hline $\mathrm{p}<0,05$ & 0 & 0,00 & 3 & 0,38 & 0 & 0,00 & 2 & 0,25 & 23 & 2,90 \\
\hline $\mathrm{p}<0,10$ & 0 & 0,00 & 6 & 0,76 & 6 & 0,76 & 13 & 1,64 & 36 & 4,55 \\
\hline $\mathrm{p}<0,15$ & 4 & 0,51 & 12 & 1,52 & 7 & 0,88 & 25 & 3,16 & 28 & 3,54 \\
\hline $\mathrm{p}<0,20$ & 6 & 0,76 & 23 & 2,90 & 13 & 1,64 & 24 & 3,03 & 20 & 2,53 \\
\hline $\mathrm{p} \geq 0,20$ & 782 & 98,74 & 748 & 94,44 & 766 & 96,72 & 728 & 91,92 & 667 & 84,22 \\
\hline Total & 792 & 100,00 & 792 & 100,00 & 792 & 100,00 & 792 & 100,00 & 792 & $\overline{100,00}$ \\
\hline
\end{tabular}




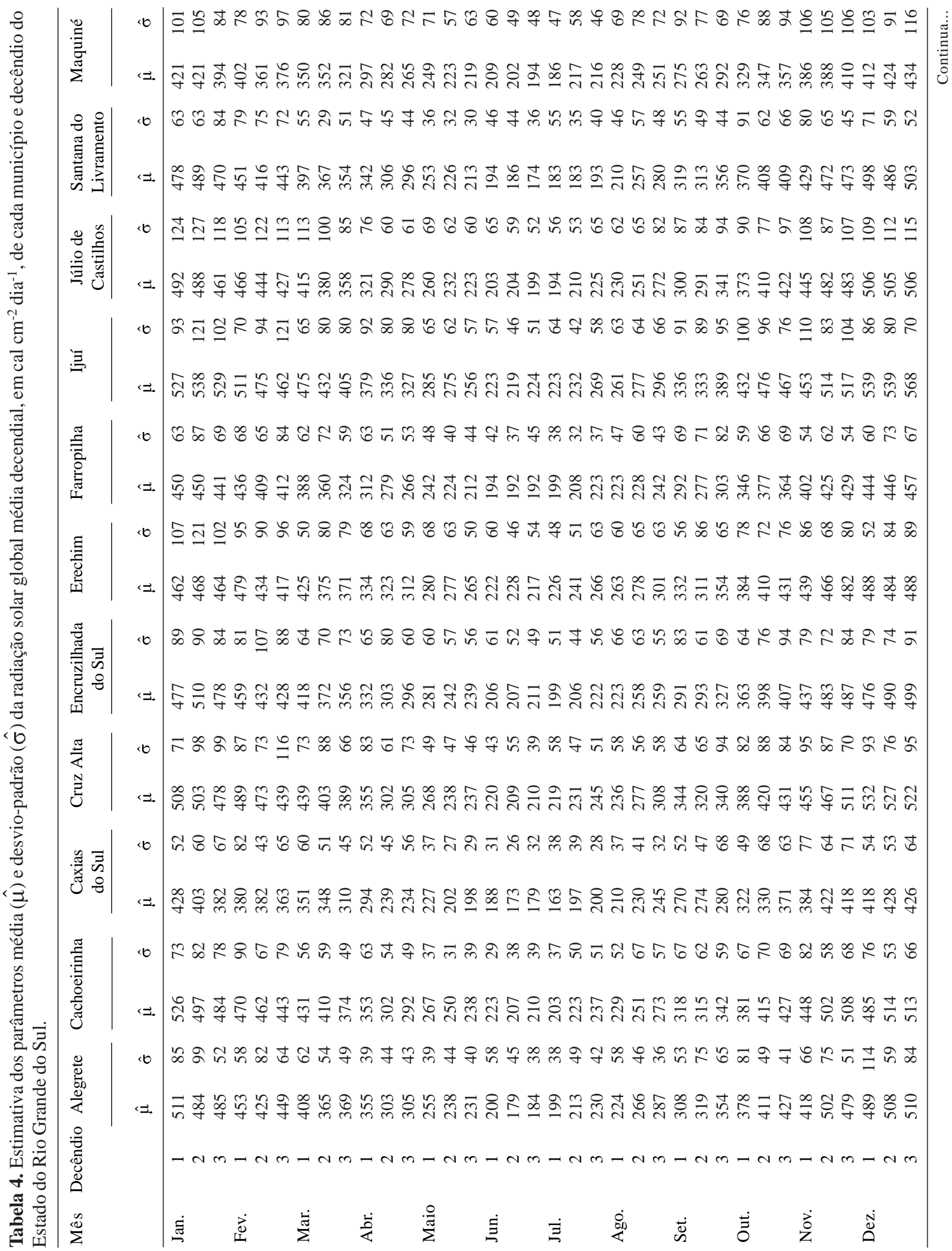




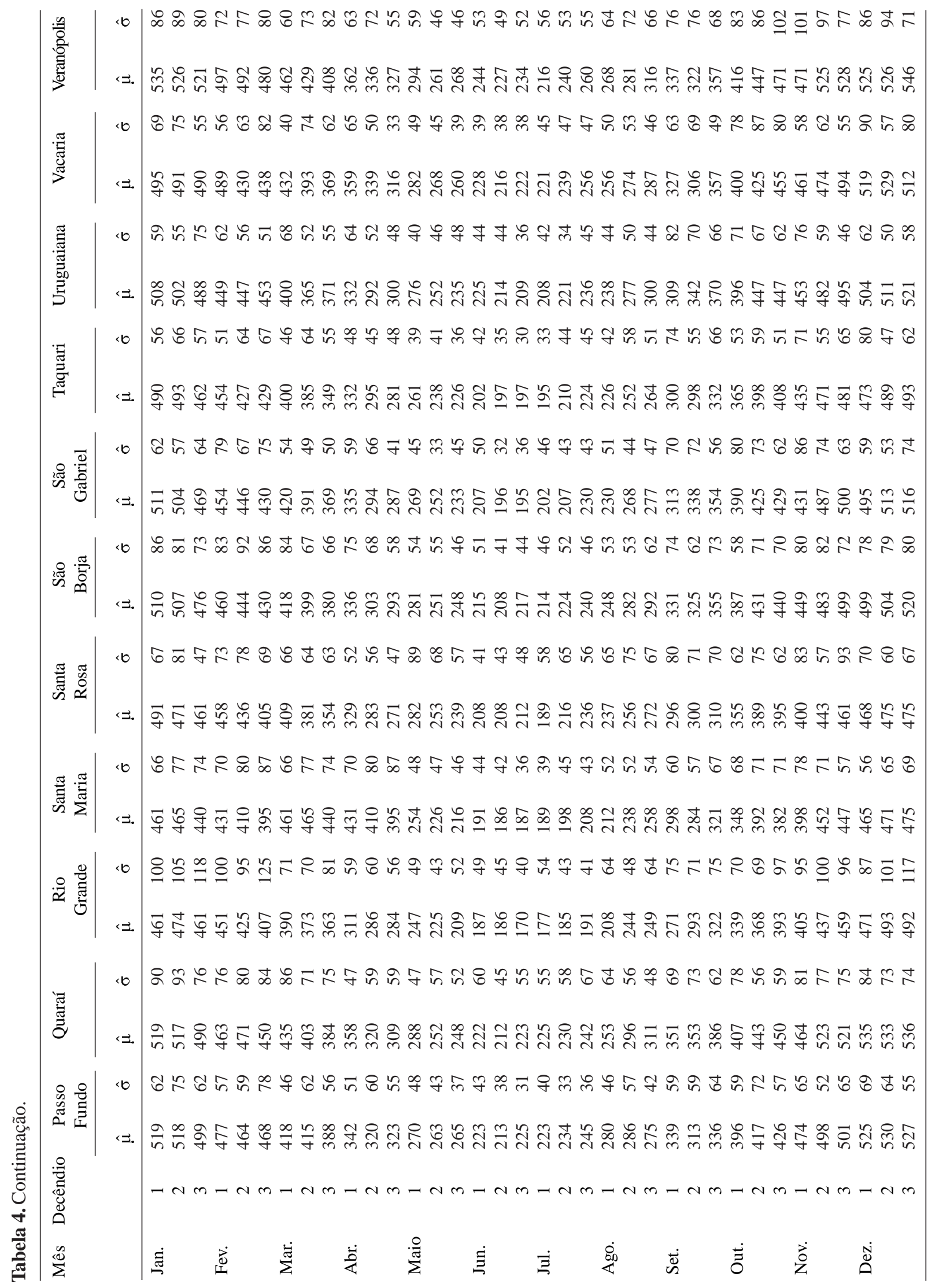



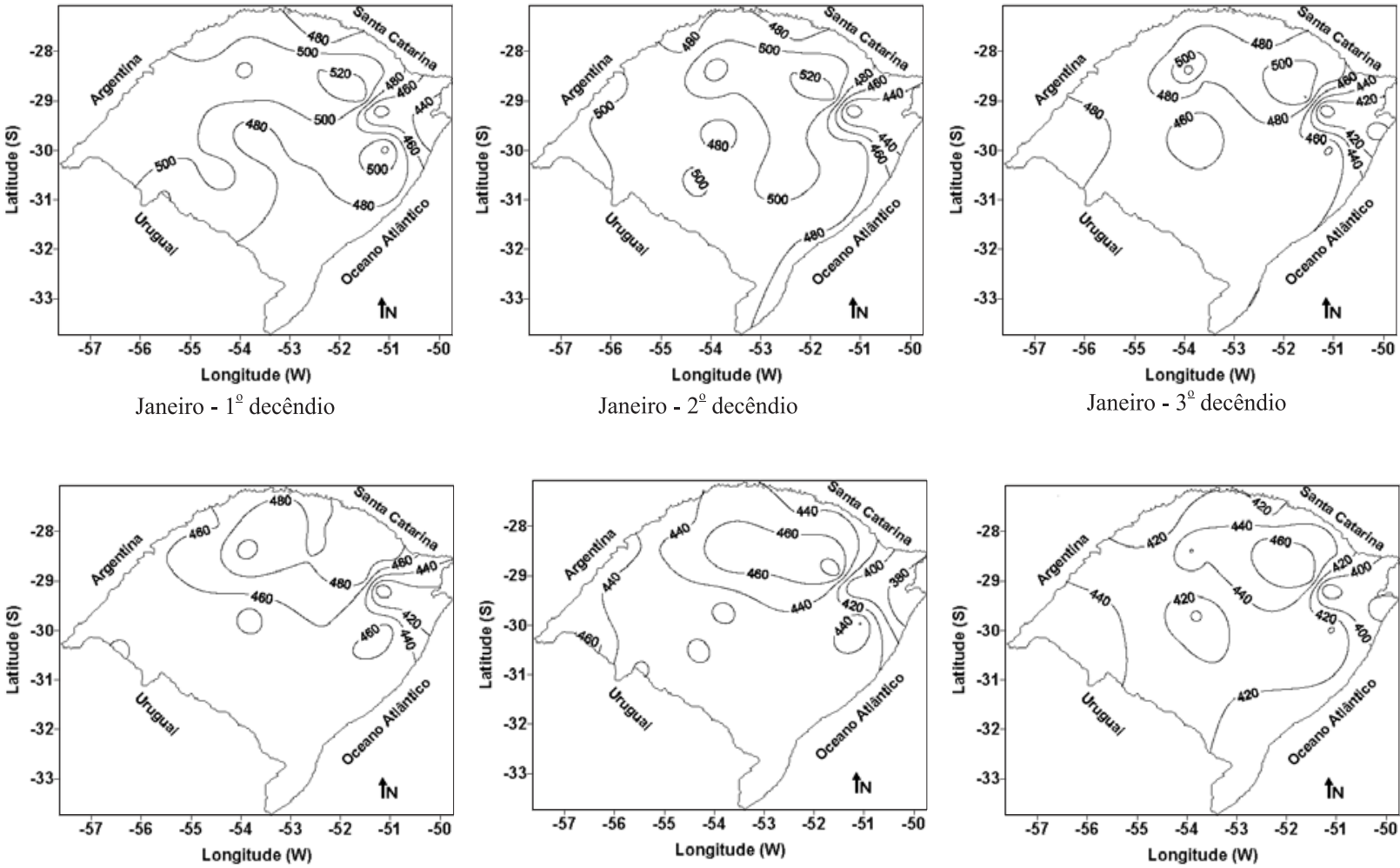

Fevereiro - $1^{\circ}$ decêndio

Fevereiro - $2^{\underline{0}}$ decêndio

Fevereiro - $3^{\underline{0}}$ decêndio
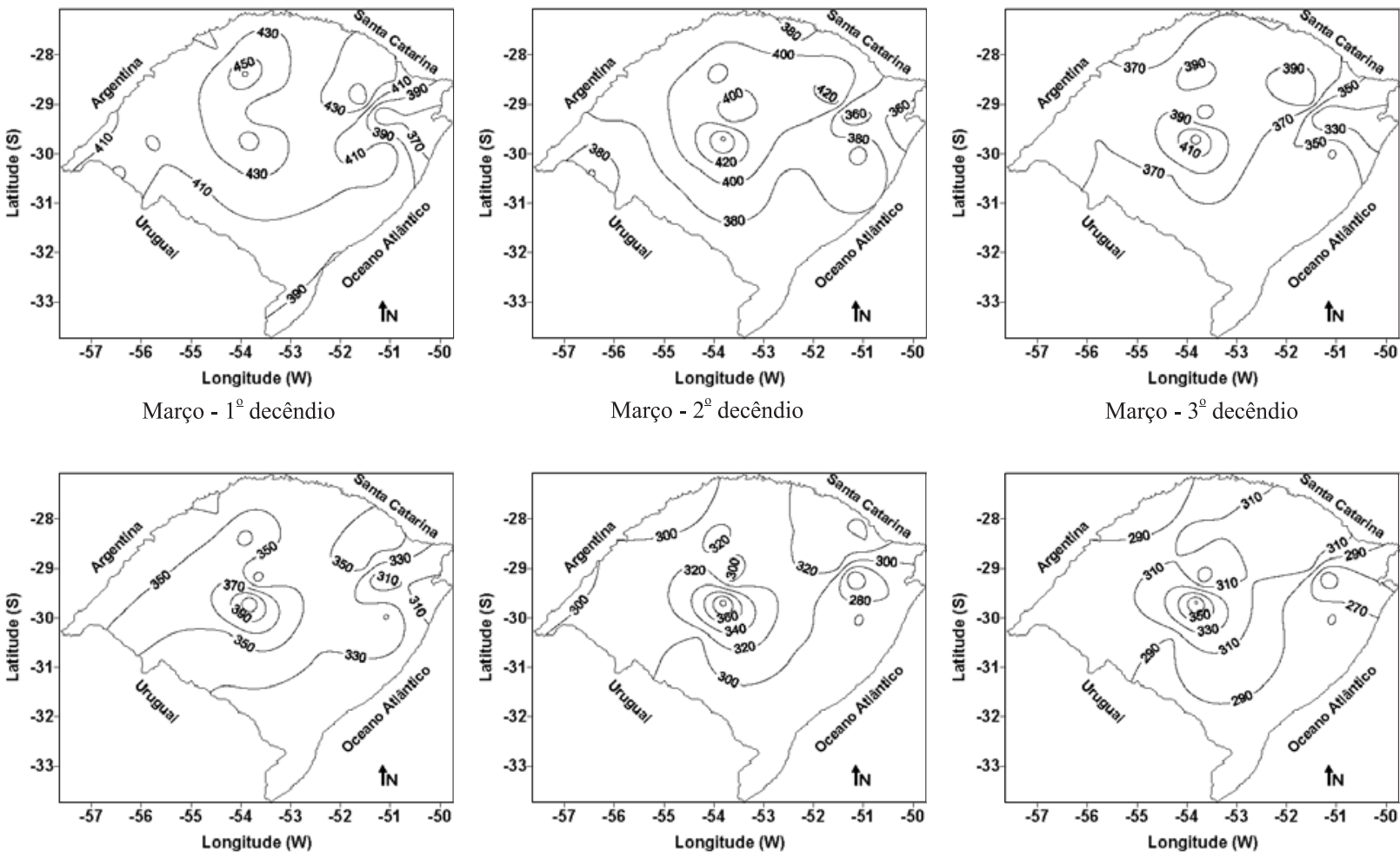

Abril - $1^{0}$ decêndio

Abril - $2^{\circ}$ decêndio

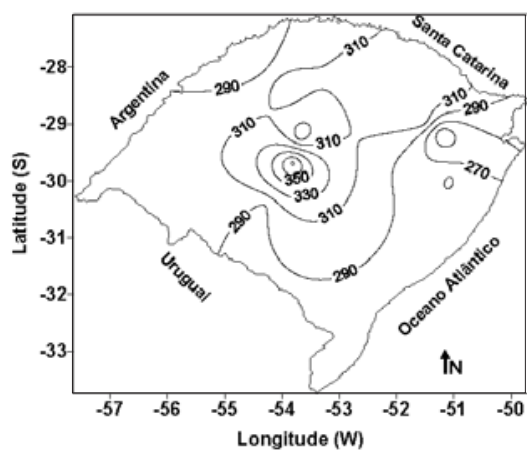

Abril - $3^{\underline{0}}$ decêndio

Figura 1. Isolinhas da radiação solar global média decendial, em cal cm${ }^{-2} \operatorname{dia}^{-1}$, de cada mês e decêndio no Estado do Rio Grande do Sul. 

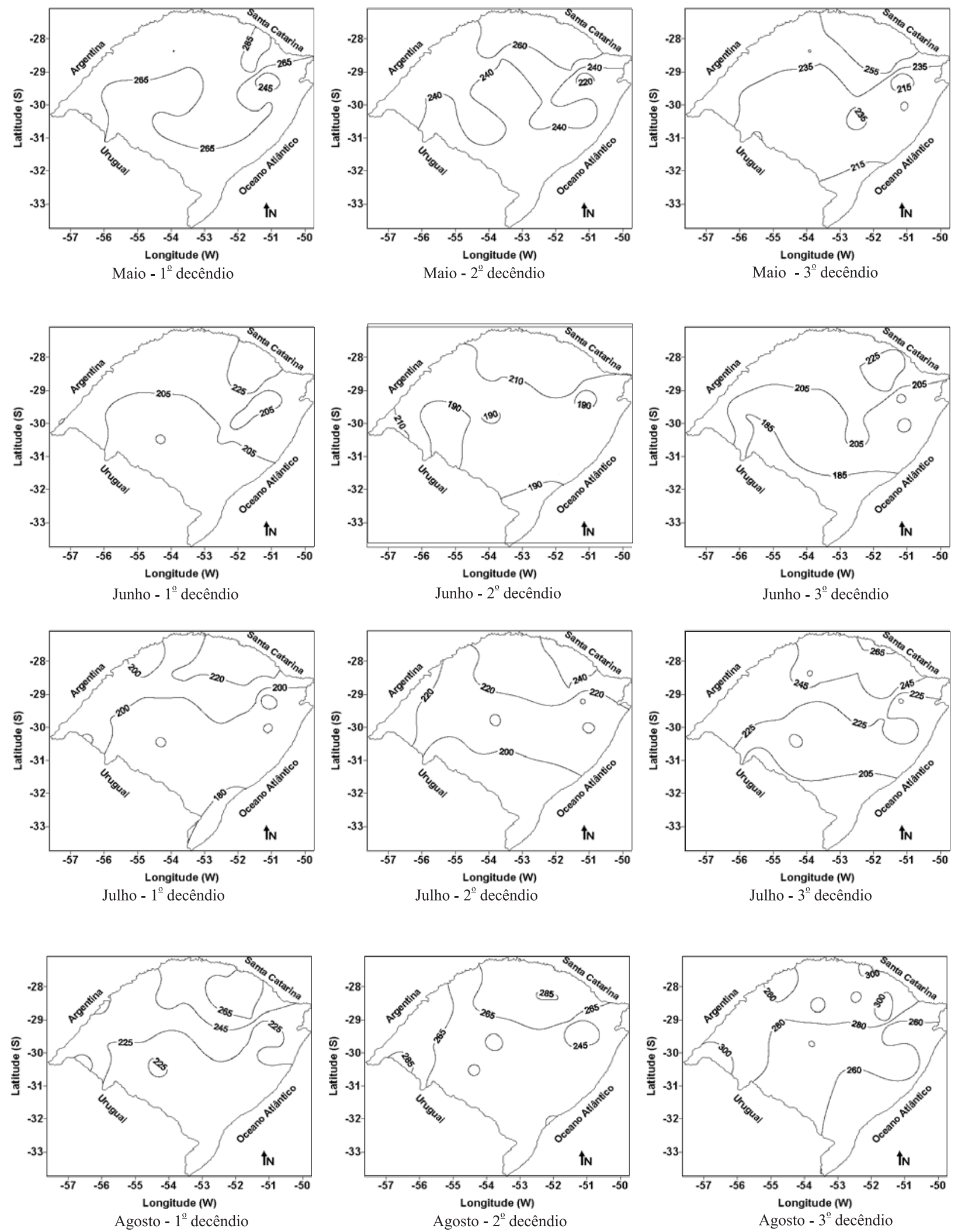

Figura 2. Isolinhas da radiação solar global média decendial, em cal cm $\mathrm{cm}^{-2} \mathrm{dia}^{-1}$, de cada mês e decêndio no Estado do Rio Grande do Sul. 

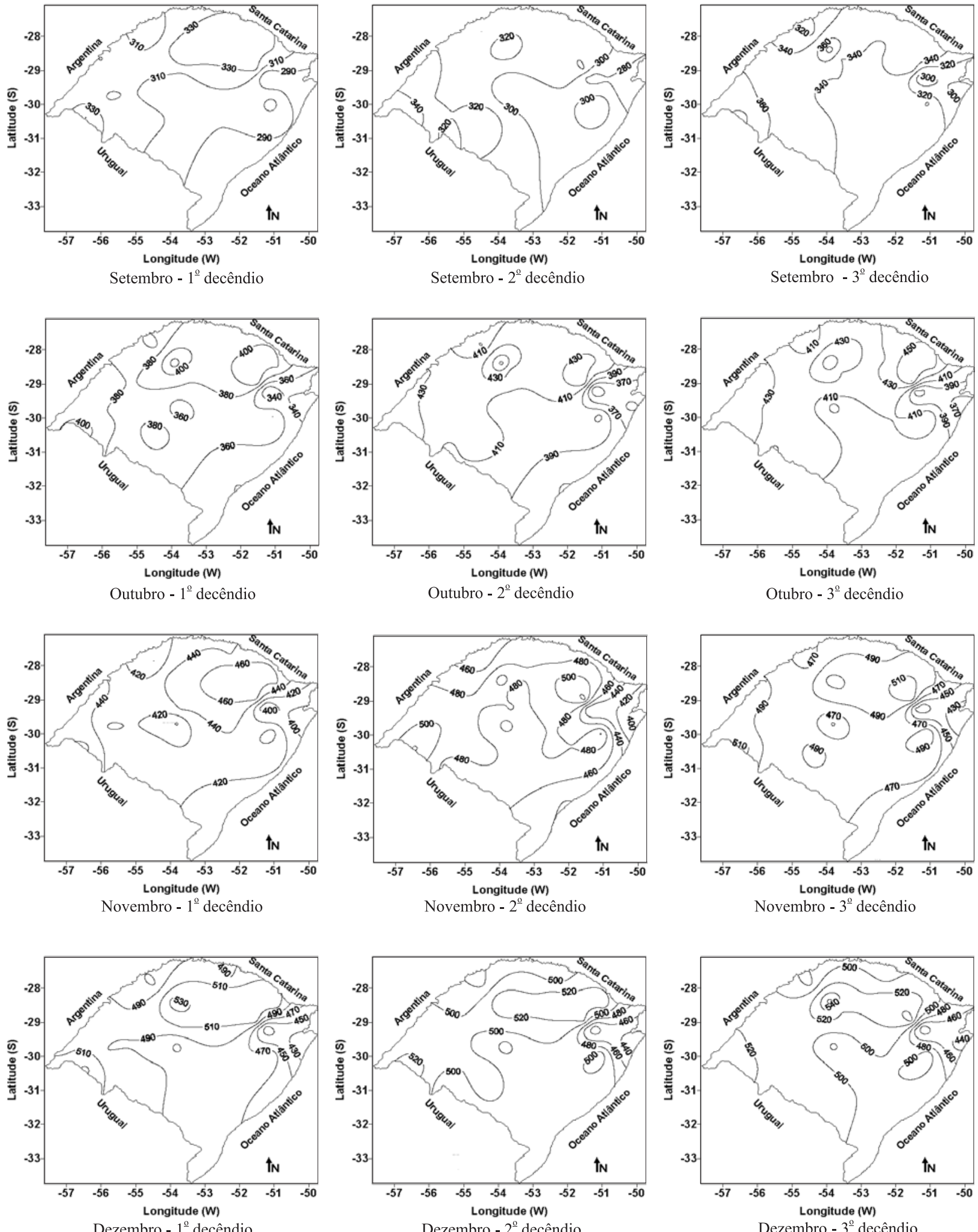

Figura 3. Isolinhas da radiação solar global média decendial, em cal cm${ }^{-2} \operatorname{dia}^{-1}$, de cada mês e decêndio no Estado do Rio Grande do Sul. 


\section{Conclusão}

Os dados de radiação solar global média decendial se ajustam às funções de distribuições de probabilidade normal, log-normal, gama, gumbel e weibull e apresentam melhor ajuste à função de distribuição de probabilidade normal.

\section{Agradecimento}

Ao conselho Nacional de Desenvolvimento Científico e Tecnológico, pela concessão de bolsa a Ronaldo Matzenauer.

\section{Referências}

ASSAD, E.D.; CASTRO, L.H.R. Análise freqüêncial da pluviometria para a estação de Sete Lagoas, MG. Pesquisa Agropecuária Brasileira, v.26, p.397-402, 1991.

ASSIS, F.N. de. Ajuste da função gama aos totais semanais de chuva de Pelotas, RS. Revista Brasileira de Agrometeorologia, v.1, p.131136, 1993.

ASSIS, F.N. de. Modelagem da ocorrência e da quantidade de chuva e de dias secos em Piracicaba, SP e Pelotas, RS, 1991. 134p.Tese (Doutorado) - Escola Superior de Agricultura Luiz de Queiroz, Piracicaba.

ASSIS, F.N. de; ARRUDA, H.V. de; PEREIRA, A.R. Aplicações de estatística à climatologia: teoria e prática. Pelotas: UFPEL, 1996. $161 \mathrm{p}$.

ÁVILA, A.M.H.; BERLATO, M.A.; SILVA, J.B.; FONTANA, D.C. Probabilidade de ocorrência de precipitação pluvial mensal igual ou maior que a evapotranspiração potencial para a estação de crescimento das culturas de primavera-verão no Estado do Rio Grande do Sul. Pesquisa Agropecuária Gaúcha, v.2, p.149-154, 1996.

BERGAMASCHI, H.; GUADAGNIN, M.R.; CARDOSO, L.S.; SILVA,M.I.G. Clima da Estação Experimental da UFRGS e região de abrangência. Porto Alegre: UFRGS, 2003. 78p.

BERLATO, M.A. Modelo de relação entre o rendimento de grãos da soja e o déficit hídrico para o Estado do Rio Grande do Sul. 1987. 93p. Tese (Doutorado) - Instituto Nacional de Pesquisas Espaciais, São José dos Campos.

BOTELHO, V.A.V.A.; MORAIS, A.R. Estimativas dos parâmetros da distribuição gama de dados pluviométricos do Município de Lavras, Estado de Minas Gerais. Ciência e Agrotecnologia, v.23, p.697706, 1999.
BURIOL, G.A.; ESTEFANEL, V.; ANDRIOLO, J.L.; MATZENAUER, R.; TAZZO, I.F. Disponibilidade de radiação solar para o cultivo do tomateiro durante o inverno no Estado do Rio Grande do Sul. Pesquisa Agropecuária Gaúcha, v.6, p113-120, 2000a.

BURIOL, G.A.; HELDWEIN, A.B.; ESTEFANEL, V.; MATZENAUER, R.; MARCON, I.A. Condições térmicas para o cultivo do pepineiro na região do baixo vale do Taquari, RS: 1. Temperaturas baixas limitantes. Pesquisa Agropecuária Gaúcha, v.6, p.205-213, 2000b.

BURIOL, G.A.; HELDWEIN, A.B.; ESTEFANEL, V.; MATZENAUER, R.; MARCON, I.A. Condições térmicas para o cultivo do pepineiro na região do baixo vale do Taquari, RS: 2. Temperatura máxima e soma térmica. Pesquisa Agropecuária Gaúcha, v.6, p.215-223, 2000c.

BURIOL, G.A.; HELDWEIN, A.B.; ESTEFANEL, V.; MATZENAUER, R.; MARCON, I.A. Probabilidade de ocorrência de valores de radiação solar prejudiciais ao cultivo do pepineiro na região do baixo vale do Taquari, RS. Pesquisa Agropecuária Gaúcha, v.7, p.97-104, 2001.

CAMPOS, H. de. Estatística experimental não-paramétrica. 4.ed. Piracicaba: Departamento de Matemática e Estatística - ESALQ, 1983. 349p.

CASTRO, R. Distribuição probabilística da freqüência de precipitação na região de Botucatu-SP. 1996. 101p. Dissertação (Mestrado) - Universidade Estadual Paulista, Botucatu.

CATALUNHA, M.J.; SEDIYAMA, G.C.; LEAL, B.G.; SOARES, C.P.B.; RIBEIRO, A. Aplicação de cinco funções densidade de probabilidade a séries de precipitação pluvial no Estado de Minas Gerais. Revista Brasileira de Agrometeorologia, v.10, p.153-162, 2002.

INSTITUTO DE PESQUISAS AGRONÔMICAS. Seção de Ecologia Agrícola. Atlas Agroclimático do Estado do Rio Grande do Sul. Porto Alegre, 1989.

MORETTIN, P.A.; BUSSAB, W.O. Estatística básica. 5.ed. São Paulo: Saraiva, 2004. 526p.

MOTA, F.S.; ROSSKOFF, J.L.C.; SILVA, J.B. Probabilidade de ocorrência de dias com temperaturas iguais ou superiores a $35^{\circ} \mathrm{C}$ no florescimento do arroz no Rio Grande do Sul. Revista Brasileira de Agrometeorologia, v.7, p.147-149, 1999.

ROSENBERG, N.J. Microclimate: the biological environmet. New York: Hohn Wiley \& Sons, 1974. 315p.

SLATER, P.N. Remote sensing, optics and optical systems. Massachussets: Addison-Wesley, 1980. 575p.

SPIEGEL, R.A.; SCHILLER, J.; SRINIVASAN, R.A. Probabilidade e estatística. 2.ed. Porto Alegre: Bookman, 2004. 398p.

Recebido em 26 de junho de 2004 e aprovado em 3 de setembro de 2004

Pesq. agropec. bras., Brasília, v.39, n.12, p.1157-1166, dez. 2004 